Article

\title{
The co-movement of Bitcoin and some African currencies - A wavelet analysis
}

\author{
Chidi U. Okonkwo ${ }^{1}$, Bright O. Osu ${ }^{2 *}$, Farid Chighoub ${ }^{3 *}$ and Ben I. Oruh ${ }^{4}$ \\ 1 Federal College of Dental Technology and Therapy Enugu, Enugu, Nigeria; chukwuoma99@yahoo.com \\ (0000-0003-2079-1877) \\ 2 Abia State University, Uturu, and MOUAU, Nigeria; osu.bright@mouau.edu.ng (0000-0003-2463-430X) \\ 3 Department of Applied Mathematics, Biskra University, Algeria; f.chighoub@univ-biskra.dz (0000-0003- \\ 2134-7989) \\ 4 Michael Opara University of Agriculture, Umudike (MOUAU), Nigeria; oruh.ben@yahoo.com \\ * Correspondence: f.chighoub@univ-biskra.dz; osu.bright@mouau.edu.ng
}

Received: date; Accepted: date; Published: 31 August 2021

\begin{abstract}
This paper investigated the co-movement between the bitcoin (BTC) and the exchange rates of some African currencies to the USD (United States Dollars) using the continuous wavelet transform (CWT) and wavelet coherence (WTC). This was done for the noisy as well as the denoised series. The CWT for the noisy series suggests high volatility for those who hold the currencies for the short term and low volatility for those who hold the currencies for a long-term period. The CWT of the denoised series suggests that volatility at low frequency is driven by noise, while volatility at a higher frequency is driven by market forces. The wavelet coherence suggests that in the presence of noise, bitcoin will be a hedge for the currencies. However, in the absence of noise, bitcoin is a haven for the Egyptian EGP, followed by the Algerian DZD, then the Nigerian NGN, and may not be a haven for the South African ZAR.
\end{abstract}

Keywords: wavelet analysis; wavelet coherence; continuous wavelet transform; co-movement-noise; exchange rate

JEL codes: C61; D53; G17

\section{Introduction}

Bitcoin is a cryptocurrency developed by Satoshi Nakamoto in 2008 (see Chohan, 2017). It was launched in 2009 as open-source software(Rotman, 2014). As an electronically created and digitally held currency, one needs a bitcoin wallet to transact with it. It can be obtained by exchange for fiat currency, goods, and services, see, e.g.(Rotman, 2014). It can also be obtained by a process called "mining". Bitcoin operates on peer-to-peer technology without a controlling body or banks. The issuance of bitcoin and the corresponding transactions are done collectively by the network. It is a platform whose source code is open-source; It is not owned or controlled by anybody. It is accessible to anyone who has access to the internet. It has certain distinct features not available in other payment platforms. This makes it appealing to users from different backgrounds (Athey et al., 2016).

Bitcoin has been compared with gold, fiat currencies, and digital currencies. It claims to be unlike gold in that it is easy to transfer, secure, verify, and granulate. It differs from fiat currencies in that its supply is limited, predictable, decentralized, and not debt-based. Unlike fiat currencies, bitcoin has a fixed quantity; it is not based on debt and has no central authority. It provides anonymity to its users, it does not freeze, and its transfer is faster and cheaper than an electronic fiat currency system.

Nakamoto published a paper titled "Bitcoin: APeer-to-Peer Electronic Cash System," where he outlined the algorithm for a peer-to-peer network that creates an electronic transaction system that 
does not rely on trust(Chohan, 2017). The amount of bitcoin in circulation is more than 18.7 million units as of the $5^{\text {th }}$ day of May 2021 and has a unit price of over USD 55 thousand.

Over time, bitcoin had increasingly found acceptance among some high-profile online retail shops as a means of payment (see Seetharaman et al., 2017). Many notable venture capitalists had invested heavily in the bitcoin project. This seems to have lent a sort of credibility to bitcoin (Seetharaman et al., 2017). One of the main reasons given for the increasing acceptance despite its very high volatility is its ability to preserve the privacy of the users. This singular property of bitcoin makes it amenable to use for money laundering, drug trafficking, and other shady deals (Jimoh \& Benjamin, 2020). Bitcoin is seen by some as a haven for financial assets, precious metals, fiat currencies, etc., see (Corbet, S. and Lucey, 2019).It is seen as a potential alternative to fiat currencies(Kristoufek, 2015).

The following advantages of bitcoin had been identified; anonymity of the identity and personal information of parties involved in transactions, immutability (records are kept permanently and cannot be changed or modified), self-sovereignty (users manage own data), transparency (distributed ledger), disintermediation (all transactions are executed by users in the trust-less environment), and collaboration (users transact with each other directly). Bitcoin is currently seen as an electronic platform for transactional payment as well as a cryptocurrency for investing, with the two being inextricably intertwined(Polasik, Michal; Piotrowska, Anna Iwona; Wisniewski, Tomasz Piotr; Kotowski, Radoslaw and Lightfoot, 2016).

Most people in African countries have seen the crypto market and particularly bitcoin as a haven to invest their wealth as a result of inflation, political and social unrest, inconsistent government policies, Central bank regulations, and so on. Others trade in foreign currencies, either in the short run or in the long run. In this work, the time-frequency approach is used to study the continuous wavelet transform of the exchange rates and the co-movement between bitcoin and the different exchange rates in the presence and the absence of noise. The exchange rates considered are the Algerian Dinar (DZD), Egyptian Pounds (EGP), Moroccan Dirham (MAD), Nigerian Naira (NGN), and South African Rand (ZAR).

As we are focused on how the correlations vary over time and at different investment perspectives, we will be utilizing wavelet analysis since it will permit the study of the features of the market in both the time and frequency dimensions. Wavelet analysis is a very powerful tool that comes in handy in studying economic and financial dynamics. The various methods for time series analysis, such as volatility and the DCC-GARCH model, can only capture changes and extract the correlation matrix only in the time domain.

From the Fourier transform, one can extract information on the frequency components of the signal. It is well known that the amplitude constant during the period the under consideration, as a result, the information regarding the time components is lost. Thus whenever breaks are present, these models are unable to capture where these changes occur. Furthermore, due to the non-stationarity produced by such breaks, transform based on Fourier approximates may not be ideal. As a result, wavelet transform is quite preferable to Fourier transform for signals that are non-stationary or only locally stationary. (Roueff \& Von Sachs, 2011). The wavelet transforms are useful in analyzing nonstationary time series at many different frequencies. (Torrence \& Compo, 1998) It is a convolution product of the time series with a wavelet.

A significant characteristic of wavelet analysis is the decomposition into the time and frequency component of economic and financial signals. The wavelet analysis usually utilizes scale, and the scale describes the frequency bands, the set of wavelet scales captures the investment horizons at which changes occur. Thus every scale depicts the dynamics of a given frequency while keeping the time dynamics. Thus wavelet analysis generates a more complex representation when compared to the time series methodology. Wavelet analysis in this way opens up some features of the market that would otherwise be hidden. This exposition implies that investors can make more informed decisions and make better investment decisions (Ramsey, 2002).

This paper investigated the relationship between bitcoin (BTC) and the exchange rate of some selected African currencies to the USD (United States Dollars). The currencies are Algerian Dinar 
(DZD), Egyptian Pounds (EGP), Moroccan Dirham (MAD), Nigerian Naira (NGN), and South African Rand (ZAR). The time series of the original exchange rate was denoted by BTC, EGP, DZD, NGN, MAD, and ZAR. Using a process called denoising, we removed the noise in the series to get the denoised series BTCd, EGPd, DZDd, NGNd, MADd, and ZARd.

The first difference of the denoised series gave the denoised, differenced series labeled BTCdd, EGPdd, DZDdd, NGNdd, MADdd, and ZARdd.This was done by plotting the continuous wavelet transform (CWT) of the original series and the denoised series. The wavelet coherence (WTC) of the original series was plotted, but we could not plot the denoised series due to a claim of stationarity, we have to difference the denoised series to be able to plot it.

The result suggests there is evidence to believe that there is co-movement between bitcoin and African currencies.

In other words, fluctuation in the bitcoin movement could be said to influence fluctuation in the exchange rate of the selected African currencies. It was seen that this co-movement could be driven by noise. However, in the absence of noise, bitcoin has a similar CWT to the Nigerian Naira. Thus in the absence of noise, we can say that bitcoin has the greatest impact on the Nigerian Naira. Having differenced the denoised series, it is easy to rank the level of coherence between bitcoin and other currencies using the color code. The plot reveals that in ascending order, the level of coherence is BTCddEGPdd, BTCddDZDdd, BTCddNGNdd, and BTCddZARdd. Thus the South African Rand (ZAR) commoves most with bitcoin followed by the Nigerian Naira (NGN) then followed by the Algerian Dinar (DZD) and then the Egyptian Pounds (EGP). The Moroccan Dirham (MAD) was indeterminate.

The remaining part of the paper consists of a literature review in section 2, methodology in section 3 , data in section 4 , results in section 5 , and discussion and conclusions in section 6 .

\section{Literature Review}

A lot of work has been done on bitcoin, wavelet, and African currencies: Bouri et al.( 2017)looked at the price returns and volatility changes in the Bitcoin market. Dyhrberg (2015) studied bitcoin, gold, and the dollar using the GARCH model. Conlon and Mcgee (2020) and Goodell and Goutte (2020) studied bitcoin and covid-19 infection and death. Jeribi and Manzli (2021) investigated if cryptocurrency could shield the Tunisian stock market during the COVID-19 pandemic. Kumah et al. (2021) considered the use of cryptocurrencies as a haven for the African financial market in the light of covid-19. Umar et al. (2020) used wavelet analysis as a tool to study the co-movement among selected commodities. Osu et al. (2020) used wavelet analysis to determine the co-movement of the stock market of the Next-11 countries.

\section{Methodology}

\subsection{Wavelet Analysis}

Wavelets are waves that grow and decay in a small time interval. They are generated from a mother wavelet $\psi(t)$, they can be expressed as a function of two parameters: the first one tell us about the location of the wavelet ( $\tau$ also called the translation parameter), and the last tells us about the analysis resolution (s also called the scaling parameter). Wavelet analysis appears to be one of the most sought-after tools for the time-frequency analysis of time series and signals. It has found applications in the fields of crude oil (Alexandridis \& Livanis, 2008), stock price (Osu et al., 2020; Okonkwo. et al., 2019), and bitcoin (Kristoufek, 2015).

Formally, wavelets are defined as:

$$
\psi_{\tau, s}(t)=\frac{1}{\sqrt{s}} \psi\left(\frac{t-\tau}{s}\right), \quad s \in \mathbb{R}^{+}, \tau \in \mathbb{R} .
$$

Where $\frac{1}{\sqrt{s}}$ is referred to as the normalization factor, which assures the comparability of wavelet transforms along with the time scales. 
A wavelet is mainly an oscillatory motion that decays rapidly. For $\psi \in \mathrm{L}^{2}(\mathbb{R})$ to be a mother wavelet, Walden and Percival[19] gave the conditions to be satisfied as; satisfying the integrability condition $\left(\int_{-\infty}^{\infty}|\psi(\mathrm{t})| \mathrm{dt}<\infty\right)$, it must have a zero mean, i.e. $\int_{-\infty}^{\infty} \psi(t) d t=0$ (oscillatory). It also must satisfy the admissibility criteria, in other words, its Fourier transform, $\mathrm{F}(x(t))=$ $\int_{-\infty}^{\infty} \psi(u) e^{-i 2 \pi u x(t)} d u$, satisfies $C_{\psi}=\int_{-\infty}^{\infty} \frac{|F(x(t))|^{2}}{x(t)} d x$ where $0<C_{\psi}<\infty$.

\subsection{Wavelet Denoising}

Wavelet denoising is the process of removing the noise from a signal to obtain a pure signal. Consider a signal $x(t)$ colored by a white noise, which can be represented as

$$
x(t)=r(t)+\varepsilon_{t} \quad(2)
$$

Where $r(t)$, is the pure signal (the marketing formation needed) and $\varepsilon_{t}$ is the random white noise that contaminates the pure signal. The signal $r(t)$ is Gaussian $r(t) \sim N\left(0, \sigma^{2}\right)$ with zero mean and variance $\sigma^{2}$. The signal $\mathrm{r}(\mathrm{t})$ is also assumed to be smooth while $\varepsilon_{t}$ is stochastic. To achieve the denoising, the signal $x(t)$ is decomposed into small groups of differing coefficients, a target mark called the threshold is chosen such that variations in the data that are less than the threshold are to be classified as noise while variations that are greater than the target mark are denoted as the pure signal.

When we take the wavelet coefficient of the colored signal $x(t)$ we have

$$
W x(t)=W r(t)+W \varepsilon_{t}
$$

Where

$W x(t)$ is the wavelet coefficient of $\mathrm{x}(\mathrm{t})$.

$W r(t)$ is the wavelet coefficient of $\mathrm{r}(\mathrm{t})$.

$W \varepsilon_{t}$ is the wavelet coefficient of $\varepsilon_{t}$.

The primary goal is to extract $W r(t)$ without the $W \varepsilon_{t}$ component. The absolute value of $W r(t)$ is assumed to be large in comparison to the variance of $W \varepsilon_{t}$. Once an estimated value of the variance is obtained, the threshold can be computed using either soft thresholding or hard thresholding. Given that:

Soft thresholding

$$
W r(t)=\left\{\begin{array}{c}
W x(t)-\operatorname{TifWx}(t)>T \\
W x(t)+T i f W x(t)<-T \\
0 \quad \text { otherwise }
\end{array}\right.
$$

Hard thresholding

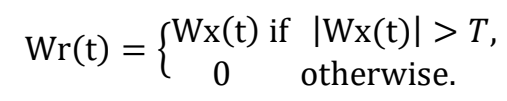

Where $T=\sigma \sqrt{2 \ln N} \sigma$ is the standard deviation.

\subsection{Continuous Wavelet Transform (CWT)}

The continuous wavelet transform (CWT) is similar to the short-time Fourier transform (STFT) in that the time and frequency domain of the given series can be extracted, but the CWT differs from STFT in that the CWT allows signals that randomly high frequency to be localized in time. The CWT can be used to extract high-frequency information within different windows in harmony with the different scales. Given a signal $x(t)$, the continuous wavelet transform can be mathematically expressed as

$$
W_{x}(u, s)=\int_{-\infty}^{\infty} x(t) \frac{1}{\sqrt{s}} \psi\left(\frac{t-u}{s}\right) d t .
$$


Where $W_{x}(u, s)$ in equation (4) is regarded as the projection of the mother wavelet on the signal. It is also regarded as the wavelet coefficient of the signal $\mathrm{x}(\mathrm{t})$. The signal $\mathrm{x}(\mathrm{t})$ can be recovered from the CWT coefficient using the mathematical formula

$$
x(t)=\frac{1}{c_{\psi}} \int_{0}^{\infty}\left[\int_{-\infty}^{\infty} W_{x}(u, s) \psi_{u, s}(t) d u\right] \frac{d s}{s^{2}}, s \neq 0
$$

This is possible if $C_{\psi}$ satisfies the admissibility condition. It is computationally costly working with continuous wavelet transform.

\subsection{Wavelet Coherency}

Wavelet Coherence is like the usual correlation coefficient, while wavelet coherence is similarly used to establish the relationship and connectedness between two signals in the time-frequency bands, the standard correlation coefficient establishes the relationship between two signals in only the time domain. Torrence and Webster gave the mathematical representation of the Wavelet Coherence of two signals say $\mathrm{x}(\mathrm{t})$ and $\mathrm{y}(\mathrm{t})$ as follows

$$
R_{x, y}^{2}(s, \tau)=\frac{\left|S\left(s^{-1} W_{x, y}(s, \tau)\right)\right|}{\sqrt{S\left(s^{-1}\left|W_{x}\right|^{2}\right) \times S\left(s^{-1}\left|W_{y}\right|^{2}\right)}}, 0 \leq R_{x, y}(s, \tau) \leq 1
$$

Where $\mathrm{S}$ in (6) is known as the smoothing operator given byS $(\mathrm{W})=S_{\text {scale }}\left(S_{\text {time }}(\mathrm{W}(\mathrm{s}))\right)$, with $S_{\text {scale }}$ representing the smoothing along the wavelet scale axis and $S_{\text {time }}$ standing for the smoothing in the time domain. The values of these operators are dependent on the choice of the mother wavelet. If the value of the wavelet coherence is close to zero, it signifies a weak correlation between the two signals. If the value is close to one, it signifies that a strong correlation exists between the two signals.

\section{Results and Discussion}

The data for this work was sourced fromwww.investing.com. The cryptocurrency bitcoin (BTC) was used as well as the Algerian Dinar (DZD), the Egyptian Pounds (EGP), the Moroccan Dirham (MAD), the Nigerian Naira (NGN) and the South African Rand (ZAR). The time series used was the original exchange rate (BTC, EGP, DZD, NGN, MAD, and ZAR). Denoising the variables, we obtain the denoised series (BTCd, EGPd, DZDd, NGNd, MADd, and ZARd). Differencing the denoised series, we got the denoised, differenced series (BTCdd, EGPdd, DZDdd, NGNdd, MADdd, and ZARdd). The data was sourced from $27 / 8 / 2012$ to $1 / 10 / 2020$, but we extracted only 1084 variables since we require the length of our variable to be of power two.

Table 1. Summary Statistics

\begin{tabular}{c|ccccccc} 
XI & Mean & Median & Minimum & maximum & Std. Dev. & Skewness & Ex Kurtosis \\
\hline NGN & 264.34 & 305.5 & 153.55 & 390.48 & 91.375 & -0.01403 & -1.8219 \\
DZD & 102.57 & 108.74 & 76.738 & 129.25 & 16.862 & -0.36194 & -1.3668 \\
EGP & 11.951 & 8.8611 & 5.8859 & 19.609 & 4.9837 & 0.12063 & -1.8775 \\
ZAR & 12.783 & 13.239 & 8.0693 & 19.248 & 2.3986 & -0.04987 & -0.51999 \\
MAD & 9.1711 & 9.3495 & 7.8811 & 10.18 & 0.57296 & -0.55869 & -0.89496 \\
BTC & 4388.4 & 3628 & 176.9 & 19476 & 4122.1 & 0.62329 & -0.60399 \\
\hline
\end{tabular}


Figure 1. Correlation Matrix

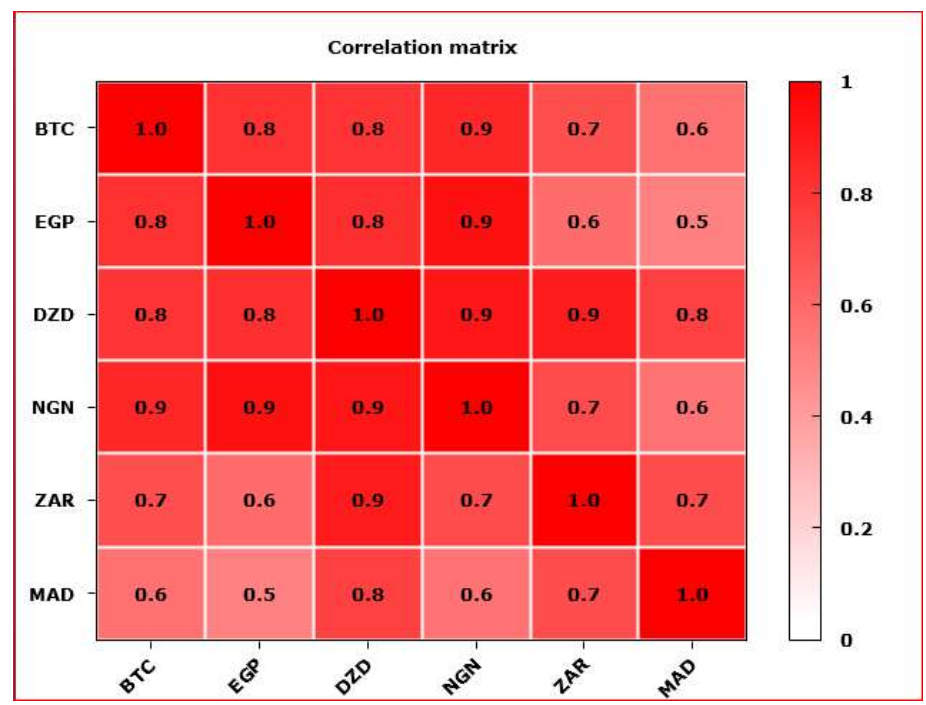

\section{Results and Discussions}

The summary statistics (Table 1) show that the BTC has very high volatility, as can be seen from the standard deviation of 4122.1. The BTC has moved from 176.9 USD/BTC to 19476 USD/BTC within the period under consideration. Of the African currencies, the NGN is the most volatile with a standard deviation of 91.375 , followed by DZD with a standard deviation of 4.9837 , while the least volatile is MAD with a standard deviation of 0.57296 .

The correlation matrix (Figure1) shows that The BTC has a very high correlation with the selected currencies. It has a correlation of 0.9 with NGN, 0.8 with EGP, and DZD. 0.7 for ZAR and 0.6 for MAD. From the correlation result, it is expected that there will be strong co-movement in the time-frequency domain.

\subsection{CWT of the Noisy Series}

The CWT (continuous wavelet transform) Figure 4 shows the variability of the currencies in the time-frequency domain. Low power is shown in blue color, while high power is shown in red, as shown in the color bar. The CWT of BTC is seen to have low energy on 0-64 on the frequency axis and $0-800$ on the time scale. The region after 800 on the time scale has a higher energy level. The right border of the zone of influence is where the higher. The CWT of EGP: all the regions outside the cone of influence have very high energy, while there is a lot of variability within the cone of influence. The CWT of DZD ZAR and NGN have a high energy level outside the cone of influence. Inside the cone of influence, there is a lot of variabilities even though the energy level is generally low. The CWT of MAD has it that the region outside the cone of influence has a high energy level. Within the cone of influence, there is a high fluctuation between low and high energy. The implication is that there is high volatility for high-frequency traders, while those low-frequency traders will experience low volatility. Simply put, volatility is high for those who hold the currencies for the short term and low for those who hold the currencies for the long term period. However, MAD has high volatility both in the long run and the short run.

\subsection{CWT of Denoised Signal}

In the absence of noise, the CWT of the currencies ( 
Figure 5) EGPd, DZDd, and ZARd are all similar. The periods below 64 on the frequency domain are at the lowest energy level, while the periods above 64 on the frequency domain have a high energy level. NGN seems to have a more similar CWT to BTC. The NGN and BTC have their energies concentrated at the right-hand corner within the cone of influence while there is a low variation of energy within the entire cone of influence. The CWT of the denoised MAD is indeterminate.

The result indicates that the low-frequency volatility is driven by noise, while volatility at a higher frequency is driven by market forces. This implies that if the African governments play their cards well, there will be more stability in its exchange rate fluctuation.

\subsection{Wavelet Coherence of Noisy and Denoised Signal}

The plot (Figure 6) presents the wavelet coherence, which portrays the co-movement in the timefrequency domain between bitcoin and the exchange rate of the selected African countries. The wavelet coherence uses a contour plot to represent the time-frequency domain where the time domain is represented by the horizontal axis, and the frequency domain is represented by the vertical axis. The color surface indicates the strength of the coherence or co-movement. The cooler color (blue) represents low coherence, while the hotter color (red) represents higher coherence. The color bar by the side is the guide on the level of intensity. The lower the co-movement,

The wavelet coherence (WTC) of BTCEGP, BTCDZD, BTCNGN, and BTCZARare all similar with low co-movement, except for few patches of high co-movement. Thus in the presence of noise, bitcoin will be a hedge for the currencies as in Figure 2.

Figure 2. Time Series Plot of the Variables
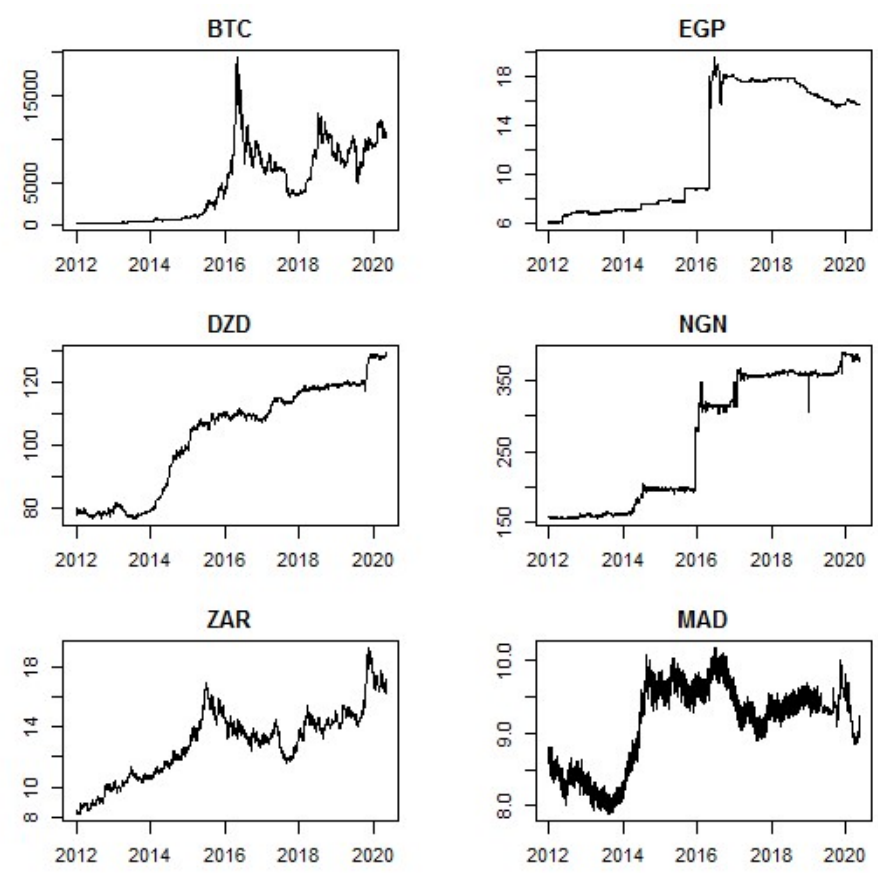

\section{Conclusion}

In this paper, we studied the relationship between the cryptocurrency bitcoin (BTC) and some selected African currencies, precisely, Algerian Dinar (DZD), Egyptian Pounds (EGP), Moroccan Dirham (MAD), Nigerian Naira (NGN), and South African Rand (ZAR). The time series used was the original exchange rate (BTC, EGP, DZD, NGN, MAD, and ZAR). Using a process called denoising, we remove the noise in the series to get the denoised series(BTCd, EGPd, DZDd, NGNd, MADd, and ZARd). Differencing the denoised series (in Figure 3), we got the denoised, differenced series (BTCdd, EGPdd, DZDdd, NGNdd, MADdd, and ZARdd). 
Figure 3. Time Series Plot of the Denoised Variables
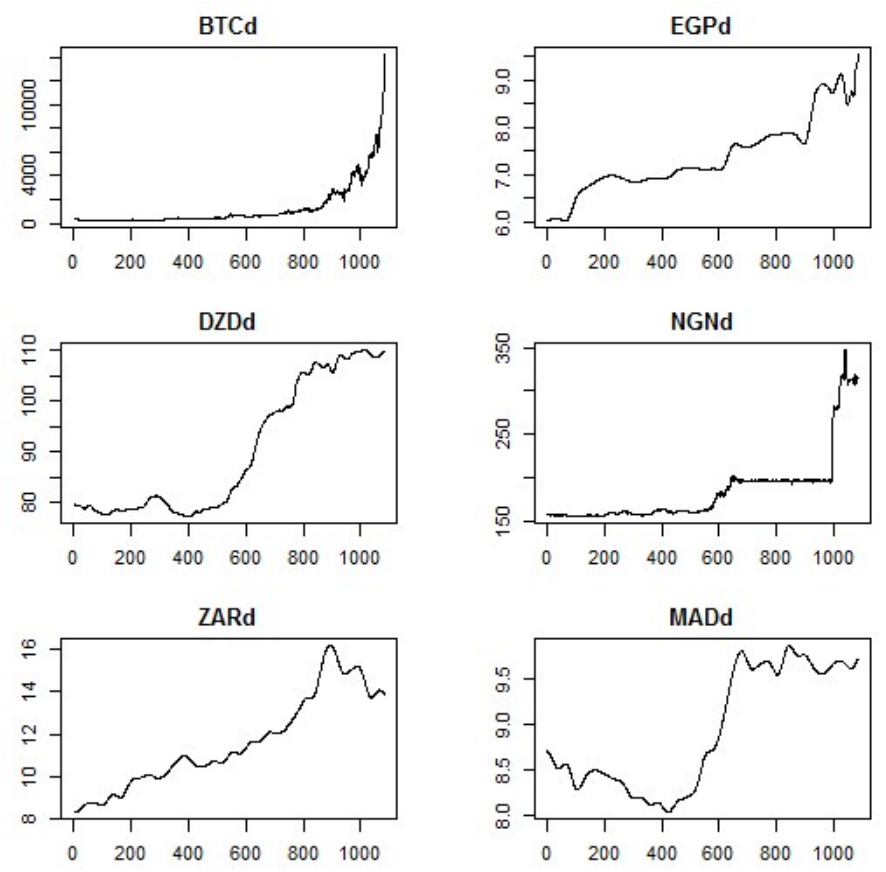

Our goal was to explore the time-frequency volatility of the selected currency and the relationship between bitcoin and the selected African currencies. This was done by plotting the CWT of the original series and the denoised series. The WTC of the original series was plotted, but we could not plot the denoised series, we must difference the denoised series to be able to plot it.

Our result shows that the CWT of BTC is seen to have low energy at low frequency and higher energy at a higher frequency. The highest energy level is concentrated at the right border of the zone of influence. The CWT of EGP shows that all the regions outside the cone of influence have very high energy while there is a lot of variability within the cone of influence. The CWT of DZD, ZAR, and NGN have high energy levels outside the cone of influence. Inside the cone of influence, there is a lot of variabilities even though the energy level is generally low. The CWT of MAD shows that the region outside the cone of influence has a high energy level. Within the cone of influence, there is a high fluctuation between low and high energy. This result seems to be at variance with the descriptive statistics. MAD had the least standard deviation implying low volatility, but it appears to have the highest volatility in the time-frequency domain, whereas BTC has the highest standard deviation but seems to have a lower variability on the time-frequency plane. This we may attribute to the presence of noise in the series, thus the need for denoising, which is a means of removing noise from a series.

After denoising, the CWT of the series shows that EGPd, DZDd, MADd, and ZARd have very low energy at the low frequencies (below 64) while all the energy is concentrated at a higher frequency. The CWT of BTCd and NGNd are similar in that there is variability over the time-frequency plane with the greater variability in the higher frequencies. This plot agrees with the standard deviation in Table 1 . The implication is that much of the variability is driven by noise. Another implication is that BTCd and NGNd are highly correlated in the absence of noise.

The wavelet coherence (WTC) of the original series here is denoted by BTCnEGPn, BTCnDZDn, BTCnNGNn, and BTCnZARn (fig.1) are all similar with high varying co-movement at the low frequencies (below 64) while there is less variability at the high frequency. The plot for BTCnMADn could not be generated. The plot reveals that so much activity occurs at a lower frequency than at a higher frequency.

Our attempt to plot the WTC of the denoised series failed over claims of non-stationarity. To correct this, the denoised series was differenced, and the CWT of the denoised and differenced series are plotted in fig a. The plot shows a uniform color. For each series, there is a single color that covers 
the entire plane with a hemispheric patch on each plot. The plot portends that the level of coherence in descending order is BTCddEGPdd, BTCddDZDdd, BTCddNGNdd, and BTCddZARdd.

There is evidence to believe that there is co-movement between bitcoin and African currencies. In other words, fluctuation in the bitcoin movement could be said to influence fluctuation in the exchange rate of the selected African currencies. It was seen that this co-movement could be driven by noise. However, in the absence of noise, bitcoin has a similar CWT to the Nigerian Naira. Having differenced the denoised series, it is easy to rank the level of coherence between bitcoin and other currencies using the color code. The plot reveals that in descending order, the level of coherence is BTCddEGPdd, BTCddDZDdd, BTCddNGNdd, and BTCddZARdd.

In summary, the CWT for the noisy series suggests high volatility for those who hold the currencies for the short term and low volatility for those who hold the currencies for a long-term period. However, MAD has high volatility both for the short term as well as the long term. The CWT of the denoised series suggests that the low-frequency volatility is driven by noise, while volatility at a higher frequency is driven by market forces. The implication is that if the right policies are implemented, there will be more stability in its exchange rate fluctuation. The wavelet coherence suggests that in the presence of noise, bitcoin will be a hedge for the currencies. However, in the absence of noise, bitcoin is a haven for the Egyptian EGP, followed by the Algerian DZD, then the Nigerian NGN, and may not be a haven for the South African ZAR.

Figure 4. CWT of the Variables

cwt of ZAR

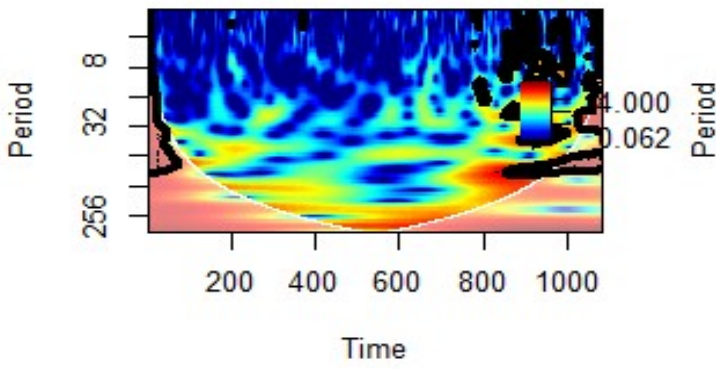

cwt of BTC

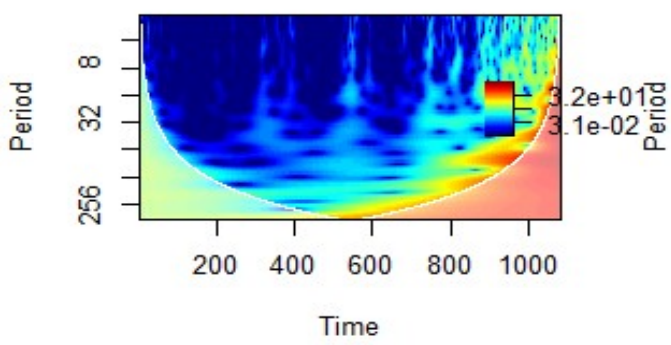

cwt of DZD

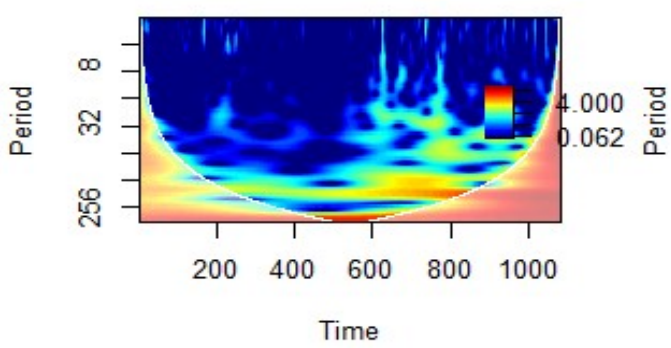

cwt of MAD

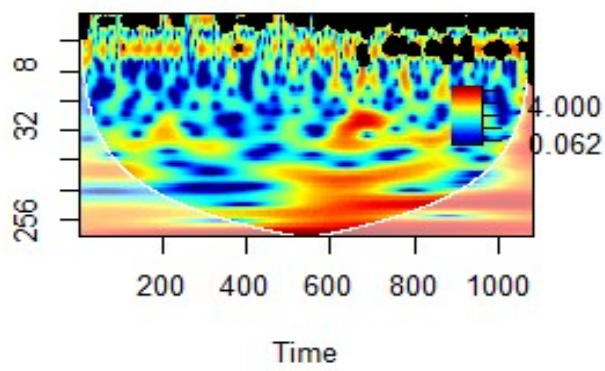

cwt of EGP

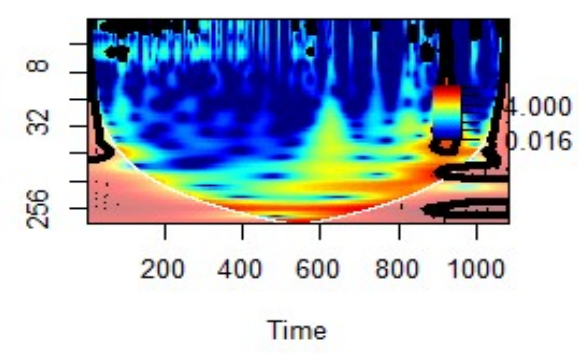

cwt of NGN

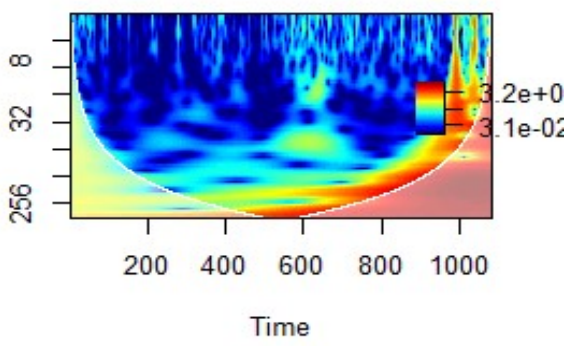


Figure 5. CWT of the denoised variables

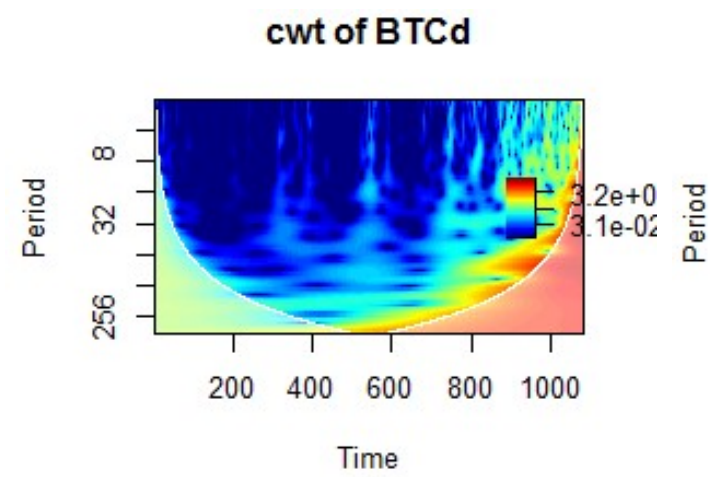

cwt of EGPd

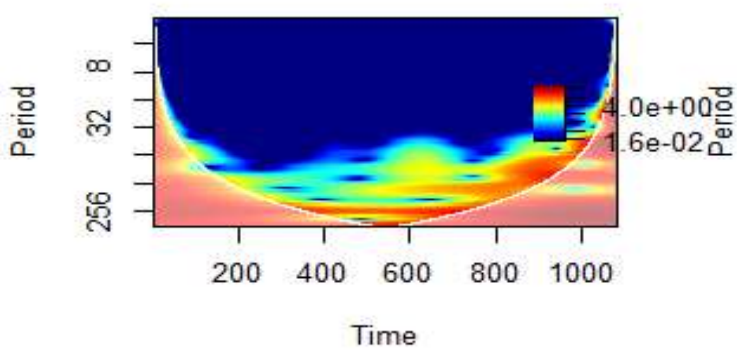

cwt of NGNd

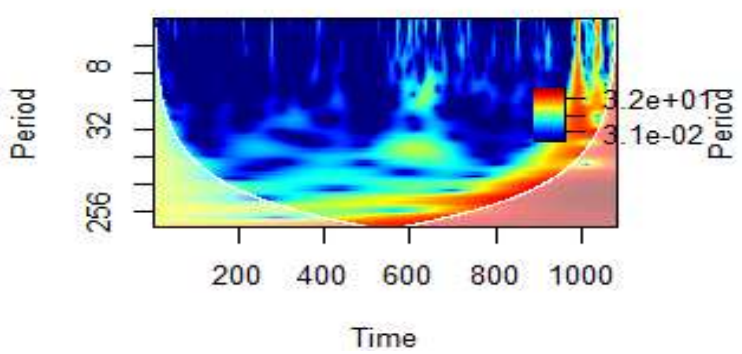

cwt of MADd

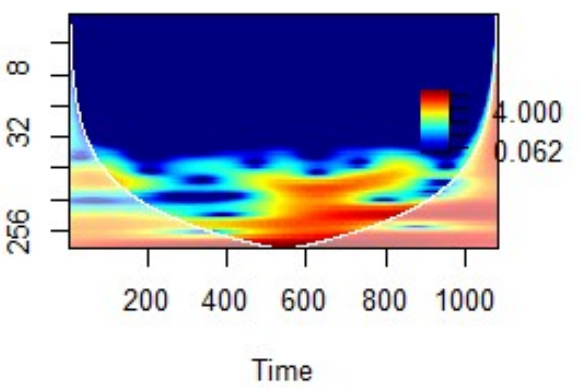

cwt of DZDd

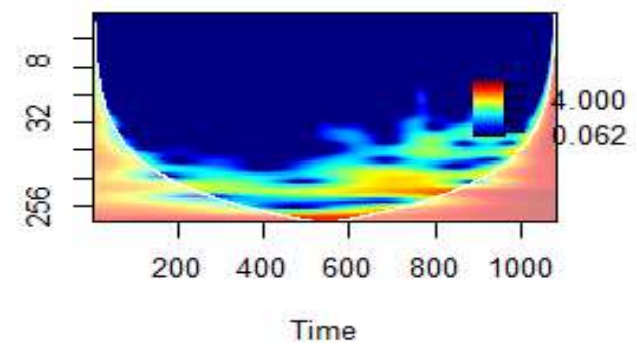

cwt of ZARd

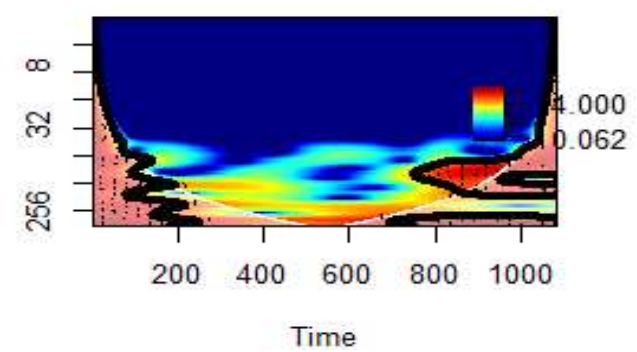

Figure 6. WTC of Denoised Variables
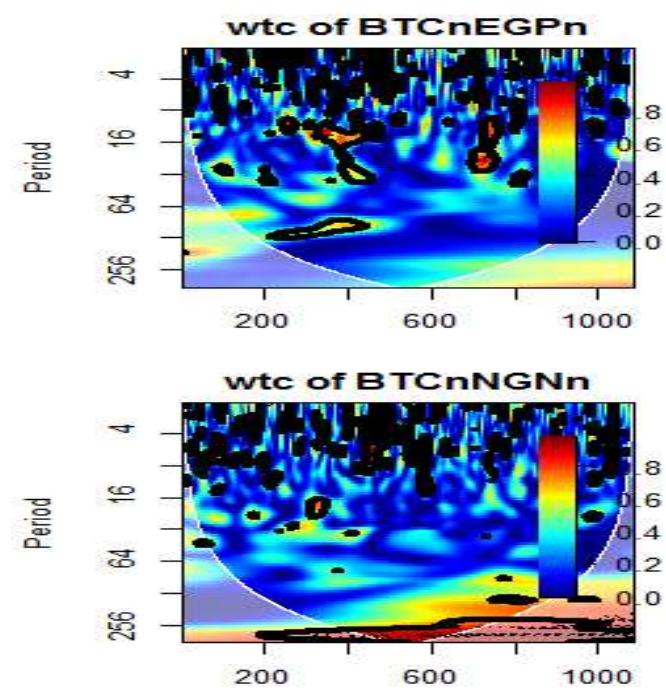
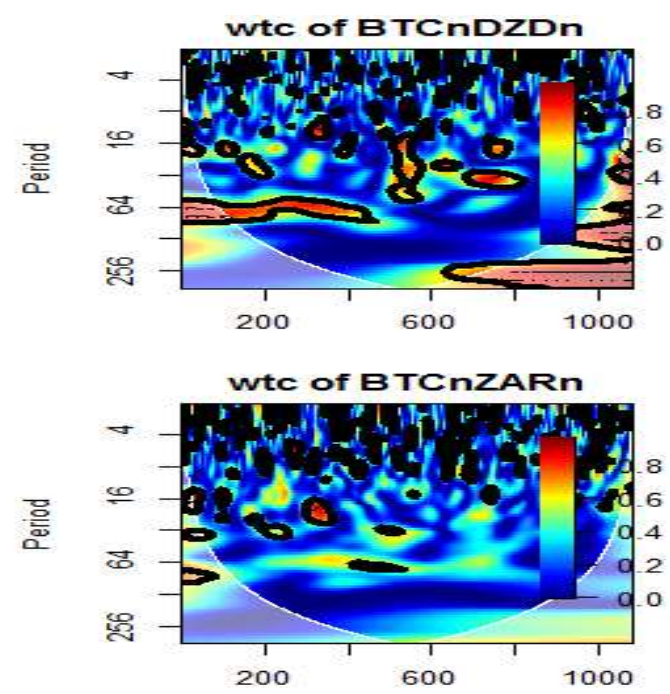
Figure 7. WTC of Denoised and Differenced Variables

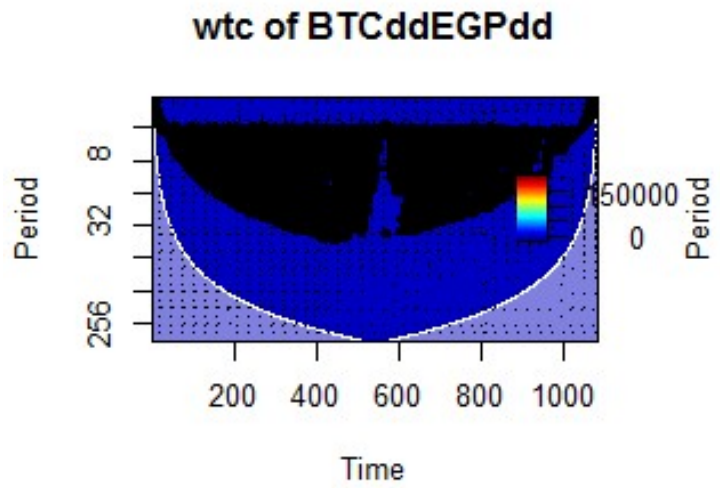

wtc of BTCddNGNdd

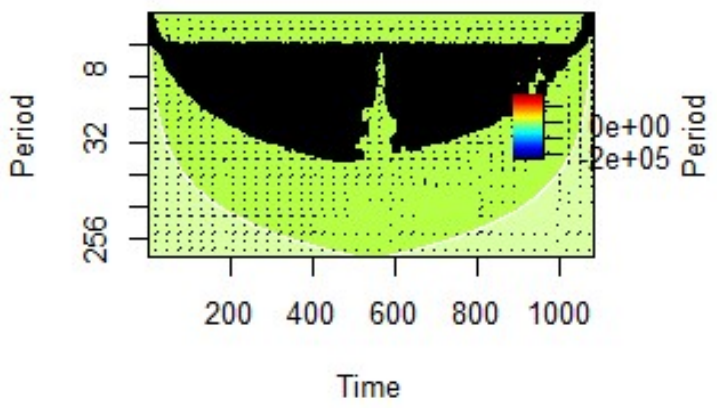

wtc of BTCddDZDdd

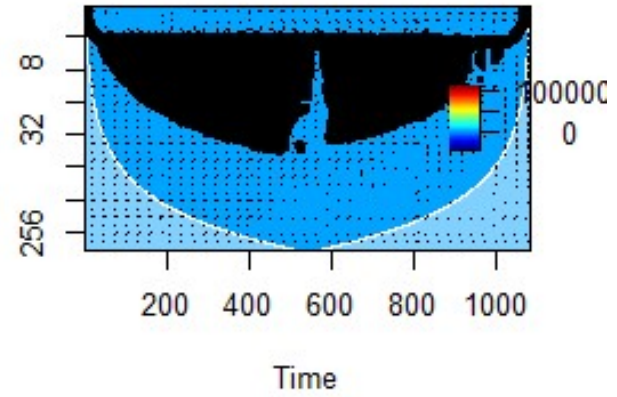

wtc of BTCddZARdd

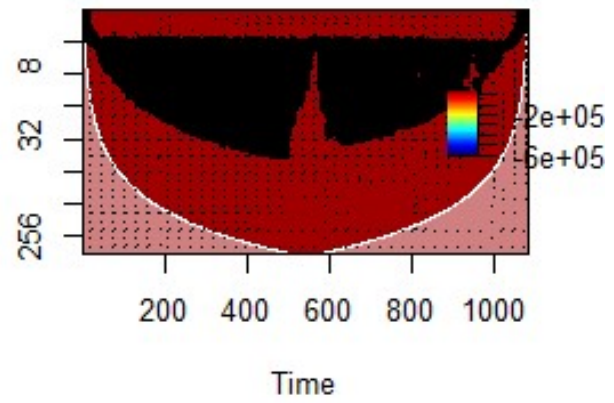

Author Contributions: Conceptualization, C.U. and B.Ó.; methodology, B.O.; software, C.U.; validation, C.U., F.C.., B.Oand B.I.; formal analysis, F.C.; investigation, F.C. and B.I.; resources, C.U..; writing-original draft preparation, C.U., F.C.., B.O and B.I.;.; writing - review and editing, , C.U., F.C.., B.O and B.I.;.; visualization, C.U.; supervision: B.O and B.I.; project administration, C.U., F.C.., B.O and B.I.

Funding: This research received no external funding.

Acknowledgments: Authors are grateful to the reviEwers of this work. Their input greatly improved this work.

Conflicts of Interest: The authors declare no conflict of interest.

\section{References}

Alexandridis, A., \& Livanis, E. (2008). Forecasting Crude Oil Prices Using Wavelet Neural Networks. Proc. 5th Student Conference of Management Science and Technology, 1-8.

Athey, S., Parashkevov, I., Sarukkai, V., \& Xia, J. (2016). Bitcoin Pricing, Adoption and Usage: Theory and Evidence (pp. 1-70).

Bouri, E., Azzi, G., \& Dyhrberg, A. H. (2017). On the return-volatility relationship in the Bitcoin market around the price crash of 2013. Economics: The Open-Access, Open-Assessment E-Journal, ISSN 1864-6042, 11(2017-2), 1-16. http://dx.doi.org/10.5018/economics-ejournal.ja.2017-2\%0AOn

Chohan, U. W. (2017). A History of Bitcoin (Notes on the 21st Century).

Conlon, T., \& Mcgee, R. (2020). Haven or risky hazard ? Bitcoin during the Covid-19 bear market. Finance Research Letters, 35(May), 101607. https://doi.org/10.1016/j.frl.2020.101607

Corbet, S. and Lucey, B. (2019). High-frequency volatility co-movements in cryptocurrency markets. Journal of International Financial Markets, Institutions and Money., ISSN 1042-4431. https://doi.org/10.1016/j.intfin.2019.05.003

Dyhrberg, A. H. (2015). Bitcoin, Gold and the Dollar - a GARCH Volatility Analysis (WP15/20). http://hdl.handle.net/10197/7168 Downloaded 
Goodell, J. W., \& Goutte, S. (2020). Co-movement of COVID-19 and Bitcoin : Evidence from wavelet coherence analysis. Finance Research Letters, 101625. https://doi.org/10.1016/j.frl.2020.101625

Jeribi, A., \& Manzli, Y. S. (2021). Can cryptocurrencies be a safe haven during the novel COVID-19 pandemic? Evidence from the Tunisian Stock Market. Journal of Research in Emerging Markets, 3(1), 14-31.

Jimoh, S. O., \& Benjamin, O. O. (2020). The Effect of Cryptocurrency Returns Volatility on Stock Prices and Exchange Rate Returns Volatility in Nigeria. ACTA UNIVERSITATIS DANUBIUS, 16(3), 200-213.

Kristoufek, L. (2015). What Are the Main Drivers of the Bitcoin Price? Evidence from Wavelet Coherence Analysis. PLoS ONE, 10(4), 1-15. https://doi.org/10.1371/journal.pone.0123923

Kumah, S. P., Abbam, D. A., Armah, R., \& Appiah-Kubi, E. (2021). African financial markets in a storm: Cryptocurrency safe havens during the COVID-19 pandemic. Journal of Research in Emerging Markets, 3(2), 60-70.

Okonkwo., C. ., Osu, B. O., Uchendu, K., \& Chibuisi, C. (2019). Wavelet Analysis of Stocks in the Nigerian Capital Market. Igerian Annals of Pure and Applied Science, 2, 176-183.

Osu, B. O., Okonkwo, C. U., Uzoma, P. U., \& Akpanibah, E. E. (2020). Wavelet analysis of the international markets : A look at the next eleven (N11 ). Scientific African, 7, 1-16. https://doi.org/10.1016/j.sciaf.2020.e00319

Polasik, Michal; Piotrowska, Anna Iwona; Wisniewski, Tomasz Piotr; Kotkowski, Radoslaw and Lightfoot, G. (2016). Price Fluctuations and the Use of Bitcoin: An Empirical Inquiry. International Journal of Electronic Commerce, 20(1), 9-49. https://doi.org/10.1080/10864415.2016.1061413

Ramsey, J. B. (2002). Wavelets in \{Economics\} and \{Finance\}: past and future. Stud. Nonlin. Dyn. Econom., 6(3), 127.

Rotman, S. (2014). Bitcoin Versus Electronic Money. OCGAP, 88164, 5-8.

Roueff, F., \& Von Sachs, R. (2011). Locally stationary long memory estimation. Stochastic Processes and Their Applications, 121(4).

Seetharaman, A., Saravanan, A. S., Patwa, N., \& Mehta, J. (2017). Impact of Bitcoin as a World Currency. Accounting and Finance Research, 6(2), 230-246. https://doi.org/10.5430/afr.v6n2p230

Torrence, C., \& Compo, G. P. (1998). A Practical Guide to Wavelet Analysis. Bulletin of the American Meteorological Society, 79(1), 61-78.

Umar, Z., Zaremba, A., \& Olson, D. (2020). Seven centuries of commodity co-movement: a wavelet analysis approach. Applied Economics Letters, 1-5.

(C) 2020 by the authors. This article is an open-access article distributed under the terms and conditions of the Creative Commons Attribution(CC BY) license (http://creativecommons.org/licenses/by/4.0/). 